\title{
Acupuncture for Diabetic Peripheral Neuropathy: A Network Meta-analysis
}

\author{
Wen-Jing Xioing, Xue Feng and Wei Chen* \\ Centre For Evidence-Based Chinese Medicine, Beijing University of Chinese Medicine, China
}

Submission: March 16, 2018; Published: August 09, 2018

*Corresponding author: Wei Chen, Centre for Evidence-Based Chinese Medicine, Beijing University of Chinese Medicine, Bei San Huan Dong Lu, Chaoyang District, 100029 Beijing, China; Email: chenweibucm@163.com

\section{Abstract}

Aim: Acupuncture therapy has been commonly used in China alone or in combination with conventional medicine to treat diabetic peripheral neuropathy (DPN), and different types of acupuncture existed. RCTs could just assess two interventions treating the same disease. When there are more than two treatments for the same disease, indirect comparisons between each treatment could not be gotten.

Objectives: To conduct a network meta-analysis to assess the potential therapeutic effects and safety of different acupuncture therapy for the treatment of DPN.

Methods: We conducted literature research from their inception to August, 2014 in the following databases: Cochrane Central Register of Controlled Trials (CENTRAL), MEDLINE, EMBASE, SinoMed, CNKI and VIP. RCTs of manual acupuncture, electroacupuncture, needle knocking acupuncture or warm needling and moxibustion for DPN were included.

Results: A total of 40 RCTs which involved 2,602 patients have been included. NMA results also indicate that compared with mecobalamin, vitamin B or no treatment, acupuncture therapy had better effect on global symptom improvement, and needle knocking acupuncture had better rank probability of effect $(60.8 \%)$.

Conclusion: NMA showed that all the included acupuncture therapies had benefit, but the needle knocking acupuncture had better rank probability than others on effect of global symptom improvement. However, due to the insufficient included studies of needle knocking acupuncture and warm needling and moxibustion, the rank probability of each intervention may still need further research to prove. In addition, the positive results should be interpreted prudently because of the high risk of bias of included trials.

Keywords: Diabetic peripheral neuropathy; Network meta-analysis; Randomized controlled trial

Abbreviations: DPN: Diabetic Peripheral Neuropathy; NMA: Network Meta-Analysis; RCT: Randomized Controlled Trial

\section{Introduction}

Diabetic peripheral neuropathy (DPN) is one of the most common chronic complications of diabetes mellitus [1]. The incidence of DPN (ranging from 30\% 90\%) is increasing over time [2], and associates with a huge disease burden in the aspect of disability and depletion of health care resources [3-5]. The only established intervention to treat DPN is strict glycemic control. Supportive therapies, including pain management and podiatric care, were also used aiming to improve quality of life and prevent chronic ulcerations [6]. However, the effectiveness is not satisfactory. Acupuncture is an alternative medicine methodology originating in ancient China. In China, acupuncture has different types. Results of our preliminary literature searching showed that among them, manual acupuncture, electroacupuncture, needle knocking acupuncture (tapping), and warm-needling and moxibustion were the most frequentlyused types of acupuncture for DPN. However, it is unclear about which acupuncture treatment is the most effective.
RCTs could just assess two interventions treating the same disease. However, when there are more than two treatments for the same disease, indirect comparisons between each treatment could not be gotten. Network meta-analysis, as a new statistical method for meta-analysis, has been commonly used to compare the efficacy and safety of multiple treatments. Therefore, we conducted a network meta-analysis of randomized clinical trials to evaluate the benefits and harms of different acupuncture interventions in patients with DPN.

\section{Methods}

We have searched the Cochrane Central Register of Controlled Trials (CENTRAL), MEDLINE, EMBASE, Chinese BioMedical CDROM (SinoMed), Chinese National Knowledge Infrastructure Database (CNKI), and VIP Chinese Science and Technique Journals Database from their inception to August, 2014. Parallel randomized controlled trials (RCTs) which assessed effects and safety of acupuncture therapy for DPN have been 
included. The acupuncture therapy was defined as the four most frequently-used types of acupuncture therapy, including manual acupuncture, electroacupuncture, needle knocking acupuncture (tapping), and warm-needling and moxibustion methods.

RevMan 5.3 and R 3.2.2 software were used to conduct Results data synthesis. Multiple treatment comparison analyses were performed through Markov-Chain-Monte-Carlo (MCMC) method. The mtc model was built and data was pooled by gemtc package. Node analysis has been performed to test the consistency of direct and indirect comparison evidence by R software.

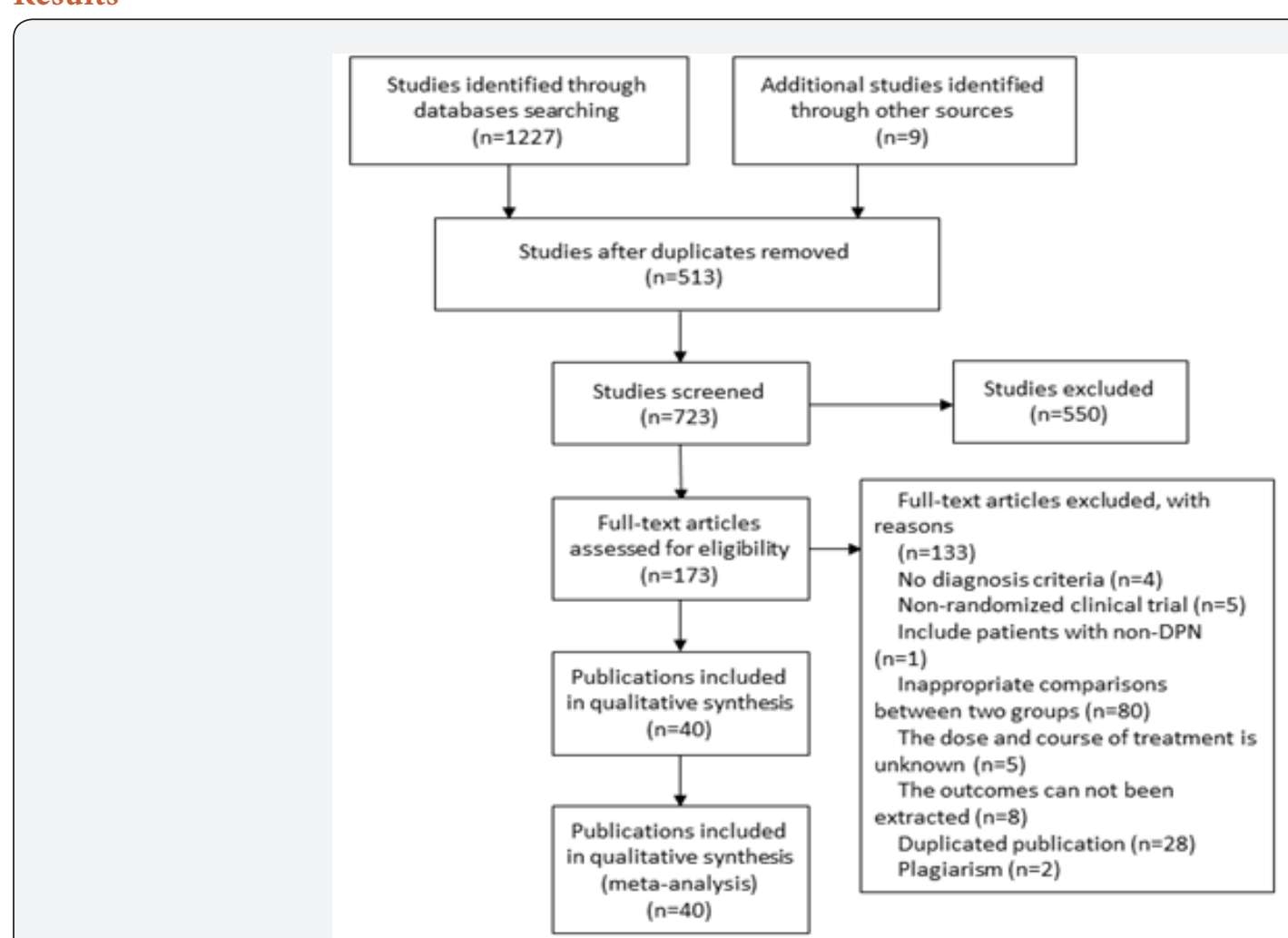

Figure 1: Flow chart.

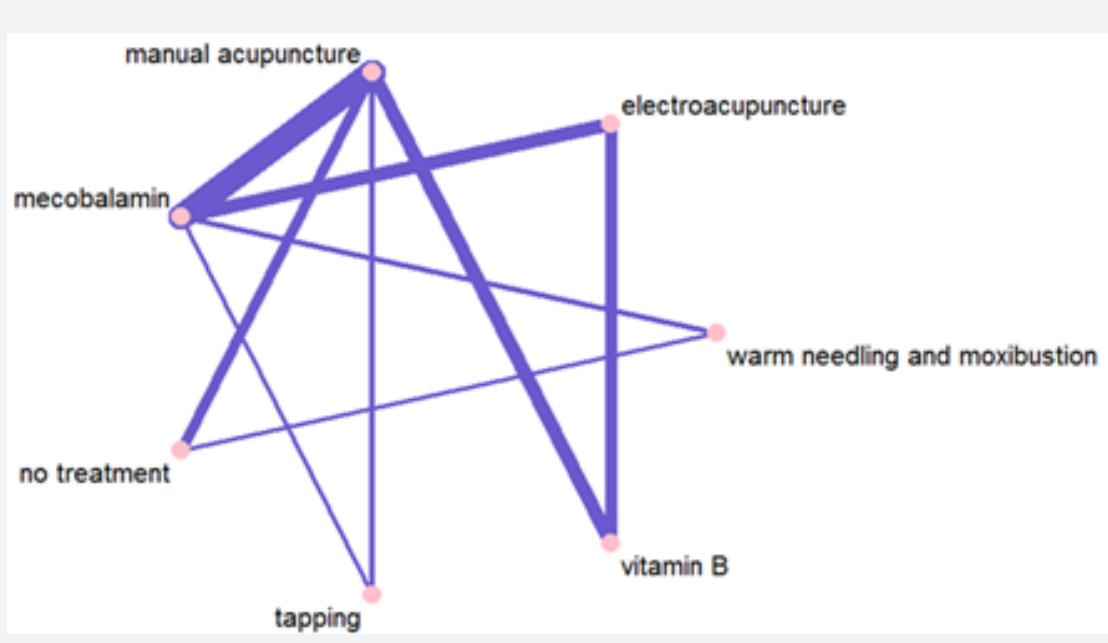

Figure 2: Network plot of included interventions by netmeta package.

A flow chart depicted the search process and study selection (Figure 1). A total of 40 RCTs involving 2,602 participants were included [7-46]. All of the included trials were performed and published in China. No trial reported patients' differentiation of symptoms. The control intervention included mecobalamin, vitamin B and no treatment. No trial used sham acupuncture or placebo as control. Basic hypoglycemic therapies including oral antidiabetic drug, insulin treatment or physical training were adopted in all included trials. The network plot of all the interventions plotted by netmeta package was shown in Figure 2. It showed that the number of trials compared manual acupuncture with mecobalamin was the most. 


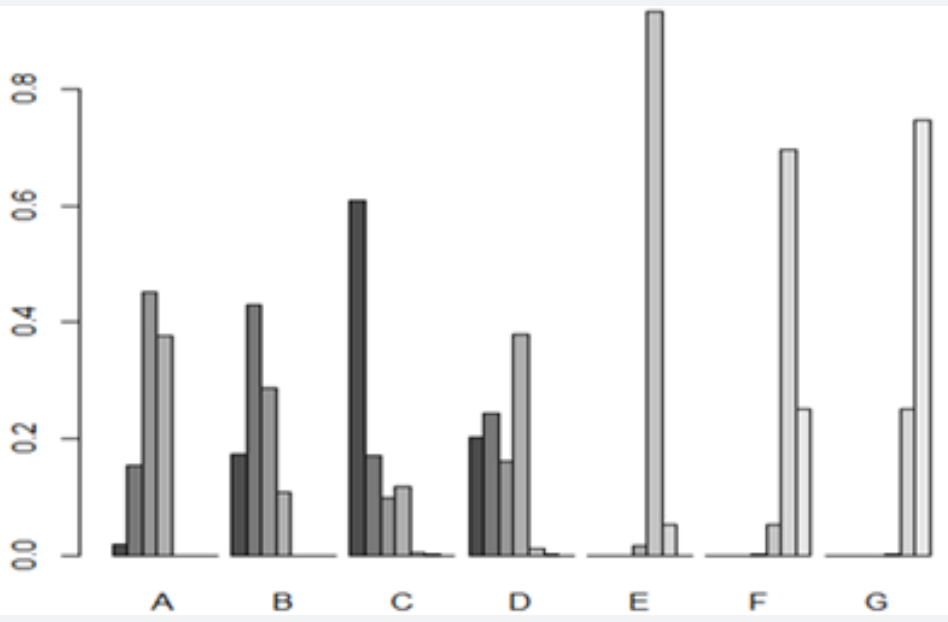

Figure 3: Rank probability plot of interventions.

(A. manual acupuncture, B. electroacupuncture, C. needle knocking acupuncture, D. warm needling and moxibustion, E. mecobalamin, F. no interventions, G. vitamin B).

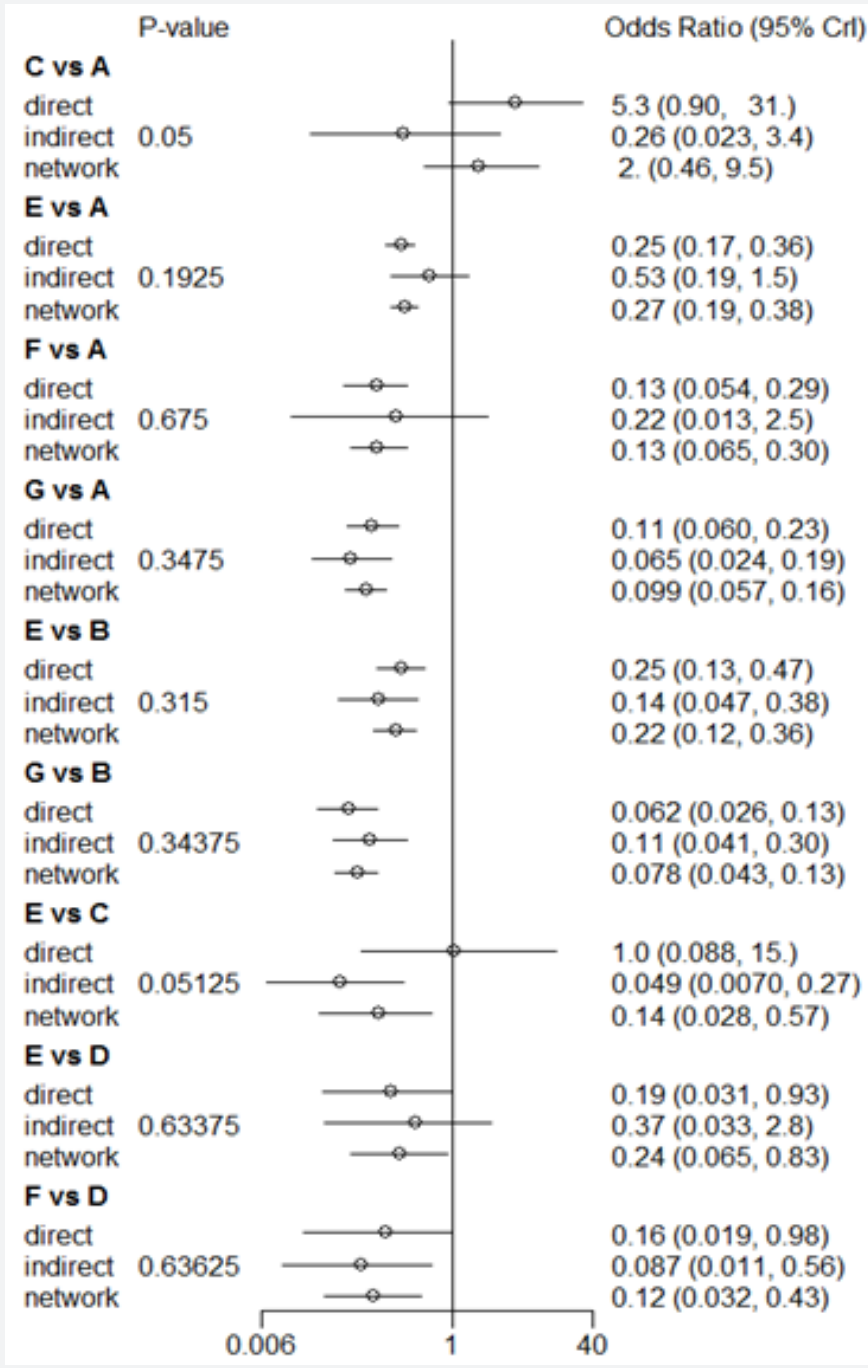

Figure 4: Forest plot of nod analyses.

(A. manual acupuncture, B. electroacupuncture, C. needle knocking acupuncture, D. warm needling and moxibustion, E. mecobalamin, F. no interventions, G. vitamin B). 
The majority of the included trials were assessed to be of general poor methodological quality according to the predefined quality assessment criteria. The NMA compared different acupuncture therapy for DPN on the outcome of global symptom improvement. We built mtc model through gemtc package, and calculated the rank probability of each intervention (Figure 3), it showed that needle knocking acupuncture has better rank probability than other interventions. We conducted the nod analyses by gemtc package, and we calculated the direct and indirect comparison results of each comparison (Figure 4). The statistic $\mathrm{P}$ value of inconsistency of each comparison was more than 0.05 , so the consistency of direct and indirect comparison was fine.

\section{Conclusion}

NMA results showed that all the included acupuncture therapies had benefit, but the needle knocking acupuncture had better rank probability than others on effect of global symptom improvement. However, due to the insufficient included studies of needle knocking acupuncture and warm needling and moxibustion, the rank probability of each intervention may still need further research to prove. In addition, the positive results should be interpreted prudently because of the high risk of bias of included trials.

\section{Acknowledgement}

This work was supported by the Chinese National Natural Science Foundation for Young Scholars (81603451). The funders had no role in study design, data collection and analysis, decision to publish, or preparation of the manuscript.

\section{Conflict of Interest}

The authors declare that there is no conflict of interests.

\section{References}

1. Lu ZQ DW (2013) Progress in diagnosis and treatment of diabetic peripheral neuropathy. Drug Evaluation 17: 35-42.

2. GM P, Y Y, P Z, Al E (2010) Clinic draft specification of traditional Chinese medicine about diabetic peripheral neuropathy. China Journal of Traditional Chinese Medicine and Pharmacy 2: 260-264.

3. Gregg E, Sorlie P, Paulose-Ram R, et al (2004) Prevalence of lowerextremity disease in the US adult population $>=40$ years of age with and without diabetes: 1999-2000 national health and nutrition examination survey. DIABETES CARE 27(7):1591-1597.

4. Savettieri G, Rocca W, Salemi G, Meneghini F, Grigoletto F, Morgante L, et al. (1993) Prevalence of diabetic neuropathy with somatic symptoms: a door-to-door survey in two Sicilian municipalities. Sicilian NeuroEpidemiologic Study (SNES) Group. NEUROLOGY 43:1115-1120.

5. Bharucha N, Bharucha A, Bharucha E (1991) Prevalence of peripheral neuropathy in the Parsi community of Bombay. NEUROLOGY 41(8): $1315-1317$

6. Poncelet AN (2003) Diabetic polyneuropathy. Risk factors, patterns of presentation, diagnosis, and treatment. Geriatrics 58(6):16-18.

7. Ren M (2007) Clinical Observation on Acupuncture for the Treatment of Diabetes and Diabetic Peripheral Neuropathy. Shenzhen Journal of Integrated Traditional Chinese and Western Medicine 4: 239-241.
8. Ren JJ (2007) Acupuncture for 30 Cases with Diabetic Peripheral Neuropathy. Journal of Clinical Acupuncture and Moxibustion 23(4): 26-27.

9. He LZ, Zhang H, Lin ZY (2009) Observation on Therapeutic Effect of Mao-acupuncture for Diabetic Peripheral Neuropathy. Symposium of the 2009 Annual Conference of China Association for Acupuncture and Moxibustion, Pp. 884-886.

10. He XJ, Wang BG, Zhan GY (2005) Clinical Effect Observation on Acupuncture for Diabetic Peripheral Neuropathy. Chinese Journal of Rehabilitation Theory and Practice 11(5): 396.

11. Yu JF, Cui ZC (2000) Clinical Study on Acupuncture Treatment of Diabetic Pathologic Changes of Peripheral Nerves. Chinese Acupuncture and Moxibustion 20(4): 203-204.

12. Liu GZ, Guo HH, Wang R, (1999) Clinical and Mechanism Research of Acupuncture for Diabetic Peripheral Neuropathy. Chinese Acupuncture \& Moxibustion 19(5): 261-264.

13. Liu TT (2005) Clinical Observation of Treatment on Diabetic Peripheral Neuropathy by Three Kinds of Acupuncture Therapies. Heilongjiang University of Chinese Medicine.

14. Ye X, Qin B, Zhang W (2013) Effect Evaluation and Investigation of its Mechanisms of Electroacupuncture for Diabetic Peripheral Neuropathy. Zhejiang Journal of Traditional Chinese Medicine 48(11): 794-796.

15. Ji XQ, Wang CM, Zhang P (2010) Effect of Spleen-stomach Regulationneedling on Nerve Conduction Activity in Patients with Diabetic Peripheral Neuropathy. Acupuncture research 35(6): 443-447.

16. Zhou XM, Liu JN, Li Y (2001) Clinical Observation on Acupuncture for 64 Cases with Diabetic Peripheral Neuropathy. Harbin Medical Journal 3: $53-54$.

17. Zhou Y, Tong J, Xu J (2014) Clinical Effect Observation on "Eightfold Method of the Sacred Tortoise" Acupuncture for 104 Cases with Diabetic Peripheral Neuropathy. Journal of Guiyang College of Traditional Chinese Medicine 36(2): 148-150.

18. Sun HQ, Hu L (2012) Clinical Observation of Tap Therapy of Magnetic Round Plum Needle Along Meridians on Patients with Diabetic Peripheral Neuropathy. Journal of Shanxi College of Traditional Chinese Medicine 13(5): 47-49.

19. Sun YZ, Xu YY, Wu D (2008) Clinical and Electrophysiological Observation on Diabetic Peripheral Neuropathy Treated by Warm Needling and Moxibustion. World Journal of Acupuncture-Moxibustion 18(2): 1-6.

20. Song BY, Hao WL (2005) Acupuncture for 22 Cases with Diabetic Peripheral Neuropathy. Journal of Sichuan of Traditional Chinese Medicine 23(7): 104-105.

21. Zhang ZQ Yao H, Chen JX (2006) Clinical Observation on Electroacupuncture for Diabetic Peripheral Neuropathy. Academic Journal of Guangzhou Medical University 34(5): 45-47.

22. Qiang BQ (2009) Acupuncture for 30 Cases with Diabetic Peripheral Neuropathy. Journal of Clinical Acupuncture and Moxibustion 25(1):25-26.

23. Xu YY (2009) Clinical Observation and Experimental Study of Effect Mechanism on Electroacupuncture Treating Diabetic Peripheral Neuropathy. Heilongjiang University of Chinese Medicine.

24. Li CH (2013) Clinical Observation on Acupuncture for Diabetic Peripheral Neuropathy. World Latest Medicine Information 22: 263264.

25. Li YF, Zheng HT, Kang EZ (2000) Clinical Research of Electroacupuncture for Diabetic Peripheral Neuropathy. Chinese Acupuncture \& Moxibustion 20(S1): 1-3. 
26. Yang ML, Lu B, Shen L (2002) Clinical Observation of Electroacupuncture for Diabetic Peripheral Neuropathy. Shaanxi Journal of Traditional Chinese Medicine 3: 261-262.

27. Shen L (2010) Efficacy evaluation of Warm Needling and Moxibustion for Diabetic Peripheral Neuropathy. GuiYang College of Traditional Chinese Medicine.

28. Wang JJ (2001) Clinical Observation of Acupuncture in Treatment of Peripheral Nervous Lesion Owing to Diabetes. Hebei Journal of Traditional Chinese Medicine 23(7): 527-529.

29. Wang CX, Sun YZ, Xu YY (2009) Clinical Observation on Treatment of 60 Cases with Diabetic Peripheral Neuropathy by Electro-acupuncture. The $17^{\text {th }}$ National Symposium on Clinical Acupuncture and Moxibustion by Clinical Branch of Chinese Association of Acupuncture-Moxibustion, Pp. 84-87.

30. Tian ZY (2013) Therapeutic Value of Acupuncture for type 2 Diabetic Peripheral Neuropathy. Asia-Pacific Traditional Medicine 9(5): 86-87.

31. Luo XT (2010) Clinical Effect Observation on Acupuncture for Symmetrical Diabetic Peripheral Polyneuropathy. Hunan University of Chinese Medicine.

32. Xu LB, Long SJ, Chen XL (2003) Effect Observation on Needle Knocking Acupuncture on Collaterals for Diabetic Peripheral Neuropathy. Modern Journal of Integrated Traditional Chinese and Western Medicine 12(6) 586-587.

33. Dong YM, Zhou J (2003) Clinical Observation on "Boosting Qi and Nourishing yin, Invigorating Blood and Unblock the Collaterals" Acupuncture for Diabetic Peripheral Neuropathy. Journal of Medical Research 32(11): 54-55.

34. Xue L, Ji XQ Yu SH (2004) Electroacupuncture for 34 Cases with Diabetic Peripheral Neuropathy. Journal of Tianjin University of Traditional Chinese Medicine 3: 152-153.

35. Yi YL, Jiang JZ, Zhang P (2009) The Effects of Oxidative Stress Status of Acupuncture the Three Main Points in Diabetic Peripheral Neuropathy Patients. Chinese Archives of Traditional Chinese Medicine 27(11) 2385-2386.
36. Yuan ZT, Zhang J, Wang HB (2008) Acupuncture for 79 Cases with Diabetic Peripheral Neuropathy. Jiangxi Journal of Traditional Chinese Medicine 39(8): 67.

37. Fei AH, Cai SC, Chen Y (2011) The Clinical Curative Effect on Diabetic Peripheral Neurophathy and Nitric Oxide with Pinprick. Journal of Clinical Acupuncture and Moxibustion 27(2): 11-12.

38. Zhao JL, Li ZR (2008) Clinical Observation on Mild-warm Moxibustion for Treatment of Diabetic Peripheral Neuropathy. Chinese Acupuncture \& Moxibustion 28(1): 13-6.

39. Zhao HL, Gao YB (2001) The Clinical Research of Treating Diabetic Peripheral Neurophathy by Acupuncture. Chinese Journal of Viral Diseases 3(3): 137-140.

40. Zhao HL, Gao X, Gao YB (2007) Clinical Observation on Effect of Acupuncture in Treating Diabetic Peripheral Neuropathy. Chinese Journal of Integrated Traditional and Western Medicine 27(4): 312314

41. Chen G (2011) Observation of Curative Effect of Acupuncture for Type 2 Diabetic Peripheral Neuropathy. Chinese Community Doctors 13(14): 180

42. Chen QY, Wu ZM (2013) Clinical Observation on 62 Cases of Diabetic Peripheral Neuritis Treated with Acupuncture. Chinese Medicine Modern Distance Education of China (12): 45-46.

43. Gu Q Yang CX (2010) Observation of Curative Effect of Combination of Acupuncture and Jia-Qi Decoction for Diabetic Peripheral Neuropathy. Journal of New Chinese Medicine 7: 88-89.

44. Ma SQ (2010) Clinical Observation on Acupuncture for 68 Cases with Diabetic Peripheral Neuropathy. Contemporary Medicine 16(34): 140141.

45. Wei RX (2008) Clinical Observation on Acupuncture and Drugs for Diabetic Peripheral Neuropathy. Nanjing University of Chinese Medicine.

46. Ma YH, Yi F (2012) "Activating Brain Function and Open the Orifices" Acupuncture for 50 Cases with Diabetic Peripheral Neuropathy. China's Naturopathy 10: 11-12.

This work is licensed under Creative

Commons Attribution 4.0 License

DOI: 10.19080/JCMAH.2018.06.555686

\section{Your next submission with Juniper Publishers will reach you the below assets}

- Quality Editorial service

- Swift Peer Review

- Reprints availability

- E-prints Service

- Manuscript Podcast for convenient understanding

- Global attainment for your research

- Manuscript accessibility in different formats

(Pdf, E-pub, Full Text, Audio)

- Unceasing customer service

Track the below URL for one-step submission https://juniperpublishers.com/online-submission.php 